\title{
A Hysteresis-free Polymer-stabilized Blue Phase Liquid Crystal
}

\author{
Yifan Liu, ${ }^{1}$ Su Xu, ${ }^{1}$ Daming $X u,{ }^{1}$ Jing Yan, ${ }^{1,2}$ and Shin-Tson $W u^{1}$ \\ ${ }^{1}$ College of Optics and Photonics, University of Central Florida, Orlando, FL 32816 \\ ${ }^{2}$ Display Center, School of Electronic Science and Engineering, Southeast University, Nanjing, China
}

\begin{abstract}
Our experimental results indicate that $U V$ curing conditions dramatically affect the electro-optic performance of a polymerstabilized blue phase liquid crystal (PS-BPLC). In an IPS cell, the $U V$ light direction, wavelength, and dosage influence the polymer network distribution, operation voltage and hysteresis. By using a longer $U V$ wavelength, we demonstrated a hysteresis-free PS-BPLC.
\end{abstract}

\section{Author Keywords}

blue phase; polymer network; UV curing; hysteresis.

\section{Introduction}

After about one decade of extensive material research and device development, the major obstacles of polymer-stabilized blue phase liquid crystal (PS-BPLC) [1,2] have been gradually overcome. For examples, the operation voltage has been reduced to $8 \mathrm{~V}$ by integrating a large Kerr constant material [3] and protruded or etched electrodes [4], while keeping high transmittance $(>80 \%)$ and submillisecond response time. High contrast ratio ( $>3000: 1)$ can be obtained by eliminating the small polarization rotation effect of BPLC $[5,6]$. Slow charging due to large LC capacitance can be accelerated by the bootstrapping driving circuitry [7]. The dawn of BPLC era has finally arrived.

However, there is one more critical issue remaining to be addressed, which is the reliability of long term operation. A PSBPLC usually contains about $10 \%$ polymers and requires UV curing process. The UV curing condition plays a key role affecting the stability and uniformity of polymer networks, which is the root cause of device reliability issues, such as hysteresis and residual birefringence. In this study, we investigate how UV exposure direction, wavelength and dosage affect the electro-optical characteristics of our IPS-based BPLC samples. By optimizing the UV exposure conditions, we demonstrated a hysteresis-free PS-BPLC.

\section{UV curing condition and mechanism}

It is commonly assumed that the polymer network of a PSBPLC is uniformly distributed across the cell gap. However, our experimental results show that this presumption is oversimplified. In the following experiments, the BPLC precursor we used consists of $84.10 \%$ HTG135200-100 $(\Delta \varepsilon \sim 86$ and

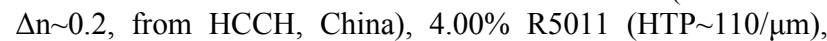
$7.11 \% \mathrm{RM} 257$ and $4.79 \% \mathrm{C} 12 \mathrm{~A}$. The precursor was filled into IPS cells, in which the electrode width/gap is $8 \mu \mathrm{m} / 12 \mu \mathrm{m}$, and cell gap is $7.5 \mu \mathrm{m}$. Two types of UV lamps were employed: Mercury lamp whose central wavelength is $\lambda=365 \mathrm{~nm}$ and UV LED lamp whose central wavelength is $\lambda=385 \mathrm{~nm}$. For each lamp, the UV light intensity and exposure time are controllable.

In the first experiment, we used the Mercury lamp with intensity $\sim 8 \mathrm{~mW} / \mathrm{cm}^{2}$ and curing time $\sim 10 \mathrm{~min}$. According to previous experiments, this dosage is sufficient to stabilize the BPLC completely. In this experiment, two samples were cured from different directions: the UV light entered the first sample from the bare substrate side (called top exposure), whereas the second sample was cured from the IPS electrode side (called bottom exposure), as depicted in Fig. 1(a). Next, we measured the voltage-dependent transmittance (VT) curves of these two samples with a He-Ne laser $(\lambda=633 \mathrm{~nm})$. Figure $1(\mathrm{~b})$ shows the measured VT curves normalized to the transmittance when the two polarizers were parallel. The high operation voltage results from the relatively large electrode gap $(12 \mu \mathrm{m})$ and small $\Delta \varepsilon(\sim 86)$ of the LC host we employed.

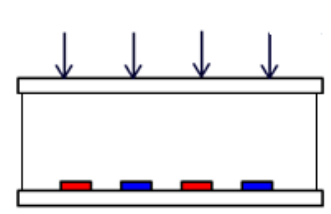

(a)

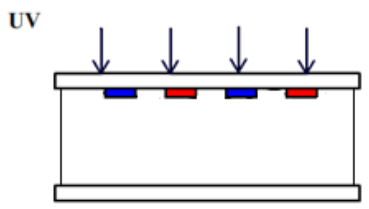

Sample 2

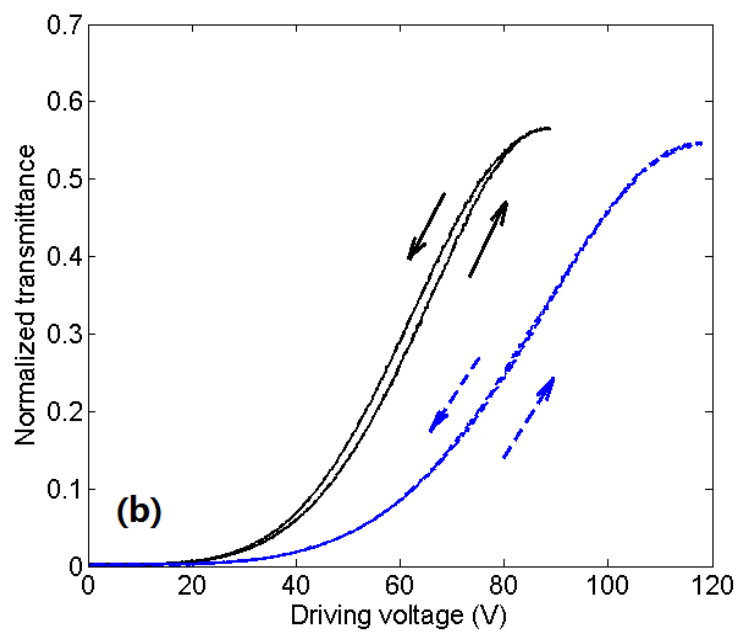

Figure 1. (a) Left: top exposure, Right: bottom exposure. (b) Measured normalized VT curves. Black solid line and blue dashed lines stand for the top exposure and bottom exposure samples by the Mercury lamp with $\lambda=365 \mathrm{~nm}$, respectively. IPS cells: electrode width $\sim \mu \mathrm{m}$, gap $\sim 12 \mu \mathrm{m}$, and cell gap $7.5 \mu \mathrm{m}$.

Figure 1(b) shows two measured VT curves: 1) black solid line is for top exposure; its $\mathrm{V}_{\text {on }} \approx 89 \mathrm{~V}$ and hysteresis $\approx 2.8 \%$. 2) Blue dashed lines represent bottom exposure. Its $\mathrm{V}_{\text {on }} \approx 118 \mathrm{~V}$, which is $\sim 32 \%$ higher, but hysteresis $(0.9 \%)$ is smaller than that of top exposure. At the first glance, this result seems contradicting to the common understanding that a lower driving voltage helps to reduce the hysteresis of a PS-BPLC [8]. But actually, our experiment and Ref. 8 address two different problems. Ref. 8 demonstrated that for the same BPLC sample, higher driving voltage causes a more noticeable hysteresis, which is attributed to the lattice distortion of the PS-BPLC. In our experiment, however, we compare two different samples cured under different UV exposure conditions, trying to reveal the details of polymerization process in BPLC. 
In order to explain our discovery, we propose the following model to illustrate the UV curing process of a BPLC sample, as drawn in Fig. 2.



(a)

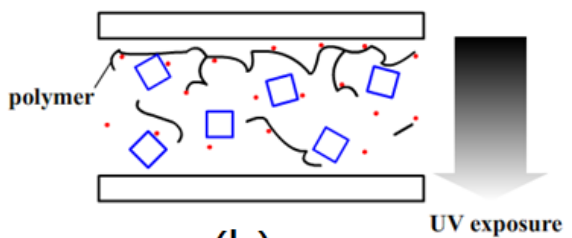

(b)

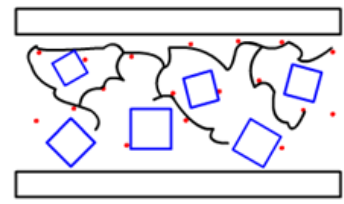

(c)

Figure 2. Polymerization process of a PS-BPLC sample: (a) The cell is filled with BPLC precursor before UV exposure. (b) BPLC sample is cured under non-uniform UV light. UV intensity is higher near the top and lower near the bottom. (c) Non-uniform polymer network of a PSBPLC sample after curing.

Before UV exposure, the LC precursor is in BP-I phase; the chiral dopant and monomers are mixed uniformly with the LC host inside the cell, as depicted in Fig. 2(a). During curing process (Fig. 2(b)), UV photons trigger the monomers to crosslink. Due to the absorption of monomers, the incident UV intensity gradually decays through the cell. Therefore, the monomers' cross-linking rate is faster near the top substrate and slower near the bottom. In this case, the monomers near the bottom substrate diffuse towards the top substrate. Thus, after UV curing process is complete (Fig. 2(c)) the polymer network is more rigid near the top substrate but looser near the bottom.

This mechanism has been used in adaptive liquid crystal lens design previously [9]. And in our case, it explains the difference between the two VT curves in Fig. 1(b). When a voltage is applied between IPS electrodes, the electric field is stronger near the electrodes, and weaker near the bare substrate side. So the liquid crystal molecular reorientation near the electrode plays a key role determining the transmittance of the device. In sample 1 (top exposure), the polymer network is looser and weaker near the electrodes. As a result, the LC molecular reorientation is easier and the driving voltage is lower. But in sample 2 (bottom exposure), the rigid polymer network covers the electrodes, so a higher driving voltage and a stronger electric field is required to reorient the LC molecules. But on the other hand, the rigid polymer network helps LC molecules to relax when the voltage is removed, so sample 2 has a smaller hysteresis.

Based on this model, it is easy to predict that if the absorption coefficient of the UV light is reduced inside the BPLC precursor, the UV light intensity distribution will be more uniform across the LC cell, and the polymer network will crosslink more uniformly inside the LC cell. To verify this hypothesis, we replaced the $365 \mathrm{~nm}$ UV lamp by a $385 \mathrm{~nm}$ UV LED lamp as our BPLC curing light source, because according to Fig. 3 below, the absorption coefficient of BPLC precursor is smaller at $385 \mathrm{~nm}$ and higher at $365 \mathrm{~nm}$.

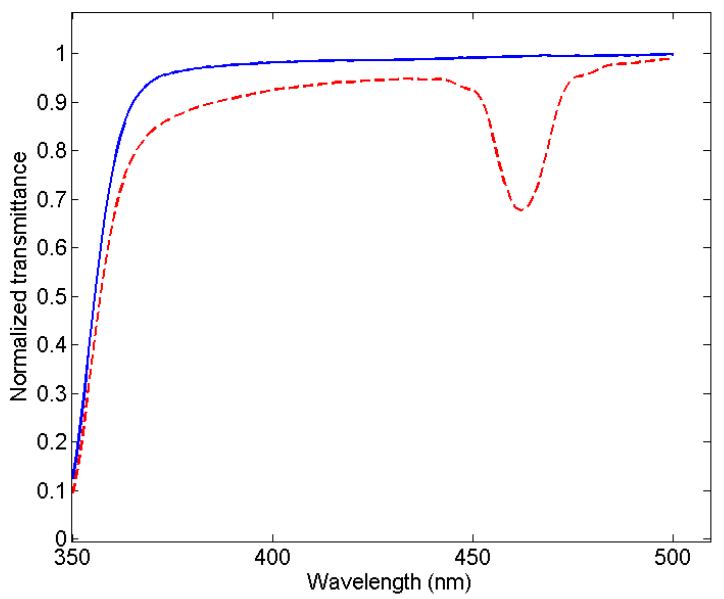

Figure 3. Measured transmission spectrum of BPLC precursor in an isotropic state (blue solid line) and BP-I state (red dashed lines).

Here we made a LC cell with $22 \mu \mathrm{m}$ cell gap sandwiched between two UV transparent $\mathrm{BaF}_{2}$ substrates. No ITO or alignment layer was coated on the substrates to avoid any absorption or scattering. The BPLC precursor mentioned above is filled into this $\mathrm{BaF}_{2}$ cell for measurement. We first heated this sample to isotropic state and measured its transmission spectrum. Then we cooled the sample to BP-I state and measured its transmission spectrum again. The measured results are normalized to the transmission spectrum of an empty $\mathrm{BaF}_{2}$ cell. In Fig. 3, the blue line represents the measured spectrum in isotropic state, where the scattering loss is negligible so that the optical loss is mainly due to the absorption of BPLC precursor. We can see that in the visible spectral region, the sample is highly transparent. But as the wavelength decreases to below $\sim 370 \mathrm{~nm}$, the transmittance declines (i.e., absorption increases) sharply. The red dashed lines in Fig. 3 represent the measured data of the sample in BP-I state. Clearly, the Bragg reflection of the BPLC sample appears at $\lambda_{\mathrm{B}} \sim 460 \mathrm{~nm}$ (which is determined by the chiral concentration in the precursor) and some light scattering is observed. From the blue curve, we find that the absorption coefficient at $\lambda=365 \mathrm{~nm}$ is $\sim 4 \mathrm{X}$ higher than that at $\lambda=385 \mathrm{~nm}$. These data indicate that under $385 \mathrm{~nm}$ UV exposure, the UV intensity will be more uniform across the LC cell. But on the other hand, we need $4 \mathrm{X}$ higher dosage at $\lambda=385 \mathrm{~nm}$ in order to fully cure the sample. It is also noticed from Fig. 3 that the absorption of the precursor in the visible region is smaller than that at $385 \mathrm{~nm}$. This explains the experimental results reported in Ref. [10], in which the PS-BPLC cured with a visible light $(>390 \mathrm{~nm})$ exhibits a more symmetric current-voltage (I-V) curve than that cured with a UV light.

According to the analysis above, we carried out another experiment using $385 \mathrm{~nm}$ UV lamp to cure the BPLC. The exposure time is extended to $40 \mathrm{~min}$, and the UV light intensity is kept at $8 \mathrm{~mW} / \mathrm{cm}^{2}$. And similarly as before, we performed the 
top and bottom exposures. Their VT curves are included in Fig. 4: the red dashed and dotted lines represent the top exposure and magenta dotted lines represent the bottom exposure (at $\lambda=385$ $\mathrm{nm})$. Both samples have almost the same $\mathrm{V}_{\text {on }}(109 \mathrm{~V}$ vs. $110 \mathrm{~V})$, indicating that our hypothesis is correct. Under $385 \mathrm{~nm}$ UV exposure, the polymer network is more uniformly distributed across the cell, so the peak voltage is irrelevant to the exposure direction of the cells. Besides, the hysteresis of top exposure sample is as low as $0.3 \%$; this "hysteresis-free" behavior is highly desirable for the accurate control of gray scales and simplification of driving circuit design.

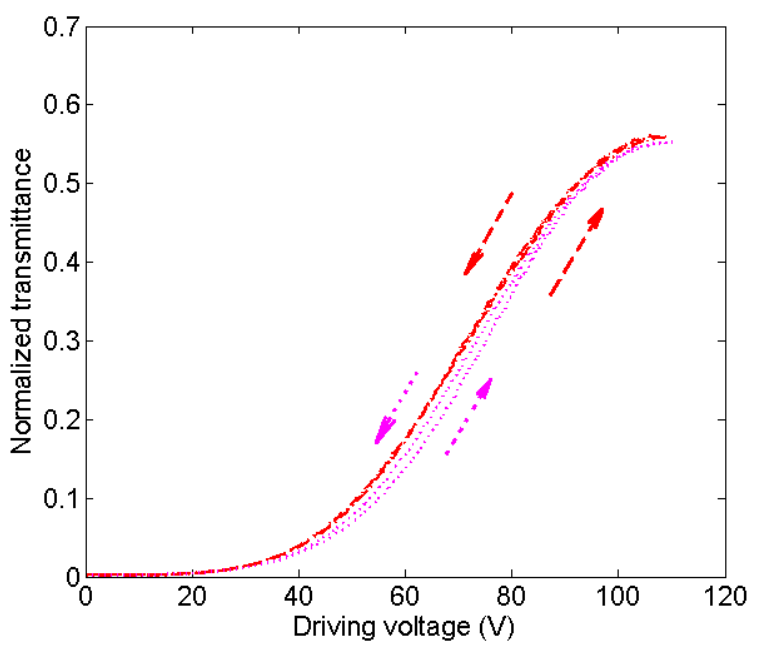

Figure 4. Measured normalized VT curves. Red dashand-dot lines and magenta dotted lines represent the top and bottom exposures at $\lambda=385 \mathrm{~nm}$, respectively.

However, it is also noticed in Fig. 4 that the bottom exposed sample demonstrates $1.7 \%$ hysteresis, which is significantly higher than the top exposed sample. We believe it is due to the absorption of the UV light by the ITO electrodes on the inner surface of the bottom substrate. According to Ref. [11], a 40nm thick ITO layer can absorb as much as $20 \%$ of incident UV light at $385 \mathrm{~nm}$. So in the bottom exposure case, the BPLC precursor under ITO electrode absorbed a lower UV dosage than the BPLC in electrode gap regions. Similarly as we analyzed above, this dosage difference may degrade the uniformity of the polymer network inside the LC cell and increase the hysteresis.

Therefore, it is concluded that to minimize the hysteresis of the PS-BPLC device, the optimal UV curing condition should be: 1) Using long wavelength UV light source; 2) Sufficient UV exposure time/dosage, and 3) Exposure from top substrate side to avoid ITO electrode absorption.

\section{Hysteresis-free PS-BPLC}

Based on the approaches discussed above, we prepared another BPLC sample with optimal curing condition. We filled the same precursor into an IPS cell and cured by a $385-\mathrm{nm}$ UV light from top side. The UV intensity was $16 \mathrm{~mW} / \mathrm{cm}^{2}$ and exposure time was $40 \mathrm{~min}$. We then measured the VT curves by ascending the applied voltage from 0 to peak voltage $\left(112 \mathrm{~V}_{\mathrm{rms}}\right)$ and then descending to 0 for three loops. The blue solid curve in Fig. 5 shows the VT curves of the three loops. The maximum hysteresis is $<0.7 \%$. And the residual transmittance after multiple driving loops remained at $0.05 \%$. Then $112 \mathrm{~V}_{\text {rms }}$ peak voltage was applied on the device for 24 hours to test the long term stability of the device. After this aging test, the V-T curve was measured again, as shown by the red dashed curve in Fig. 5. This V-T curve is slightly shifted away from the original blue curve, but the dark state light leakage is the same as before, implying that no residual transmittance was induced. The hysteresis after aging test is still $0.6 \%$, similar to the value before aging test. These results prove that this PS-BPLC sample has quite good stability. Although the driving voltage of this sample is still high compared to other reported BPLC materials $[12,13]$, our main objective here is to demonstrate a polymerization process which can lead to hysteresis-free device with good long term stability.

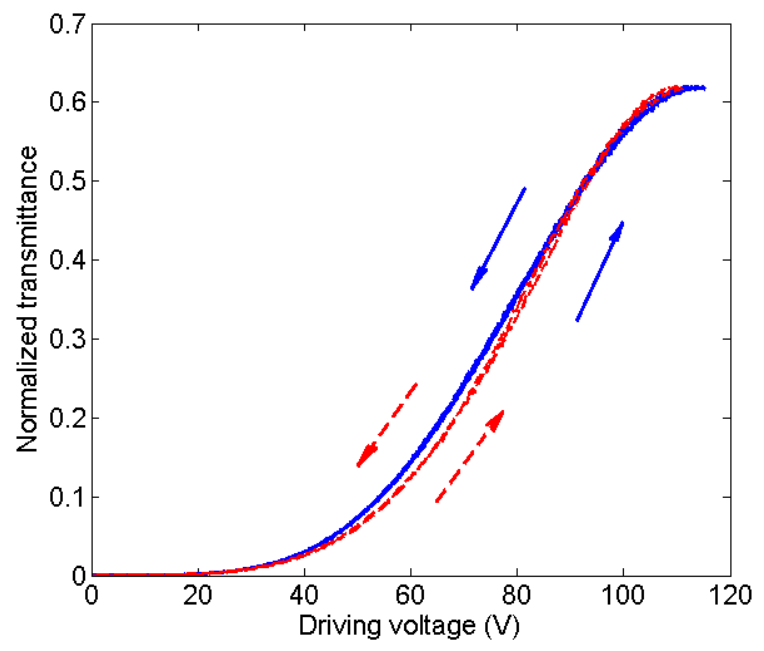

Figure 5. V-T loops of BPLC IPS sample cured under optimized curing condition. Blue solid curve is the threeloop driving cycle. Red dashed curves are the V-T curve after 24 hours of aging test.

\section{Conclusion}

We investigate the polymerization process of PS-BPLC under different UV exposure conditions. By controlling the UV exposure direction, wavelength and dosage, we demonstrated a hysteresis-free PS-BPLC with $<1 \%$ hysteresis. This result could improve the long-term stability of the BPLC devices, and simplify the driving circuit design of BPLC display system.

\section{References}

[1] H. Kikuchi, M. Yokota, Y. Hisakado, H. Yang, and T. Kajiyama, "Polymer-stabilized liquid crystal blue phases," Nat. Mater. 1, 64-68 (2002).

[2] Z. Ge, S. Gauza, M. Jiao, H. Xianyu, and S. T. Wu, "Electro-optics of polymer-stabilized blue phase liquid crystal displays," Appl. Phys. Lett. 94, 101104 (2009).

[3] Y. Chen, D. Xu, S.T. Wu, S. Yamamoto, and Y. Haseba, "A low voltage and submillisecond-response polymerstabilized blue phase liquid crystal," Appl. Phys. Lett. 102, 141116 (2013).

[4] D. Xu, Y. Chen, Y. Liu, and S. T. Wu, "Refraction effect in an in-plane-switching blue phase liquid crystal cell," Opt. Express 21, 24721-24735 (2013).

[5] Y. Liu, Y. F. Lan, H. Zhang, R. Zhu, D. Xu, C.Y. Tsai, J.K. $\mathrm{Lu}$, N. Sugiura, Y.C. Lin and S. T. Wu, "Optical rotatory 
power of polymer-stabilized blue phase liquid crystals," Appl. Phys. Lett. 102, 131102 (2013).

[6] Y. Liu, Y. F. Lan, Q. Hong and S.-T. Wu, "Compensation film designs for high contrast wide-view blue phase liquid crystal displays," J. Display Technol. 10, 3-6 (2014).

[7] C. D. Tu, C.L. Lin, J. Yan, Y. Chen, P.C. Lai and S. T. Wu, "Driving scheme using bootstrapping method for bluephase LCDs," J. Display Technol. 9, 3-6 (2013).

[8] L. Rao, J. Yan, S. T. Wu, Y. C. Lai, Y. H. Chiu, H. Y. Chen, C. C. Liang, C. M. Wu, P. J. Hsieh, S. H. Liu and K. L. Cheng, "Critical field for a hysteresis-free blue-phase liquid crystal device," J. Display Technol. 7, 627-629 (2011).

[9] H. W. Ren and S. T. Wu, "Tunable electronic lens using polymer network liquid crystals" App. Phys. Lett. 82, 2224 (2003).
[10] S. I. Yamamoto, T. Iwata, Y. Haseba, D.U. Cho, S. W. Choi, H. Higuchi, and H. Kikuchi, "Improvement of electro-optical properties on polymer-stabilised optical optically isotropic liquid crystal," Liq. Cryst. 39, 487-491 (2012).

[11] I. C. Khoo and S. T. Wu, "Optics and Nonlinear Optics of Liquid Crystals" (World Scientific, 1993).

[12] L. Rao, Z. Ge, S. T. Wu, and S. H. Lee, "Low voltage bluephase liquid crystal displays," Appl. Phys. Lett. 95, 231101 (2009).

[13] Y. Chen, D. Xu, S.T. Wu, S. Yamamoto, and Y. Haseba, "A low voltage and submillisecond-response polymerstabilized blue phase liquid crystal," Appl. Phys. Lett. 102, 141116 (2013). 